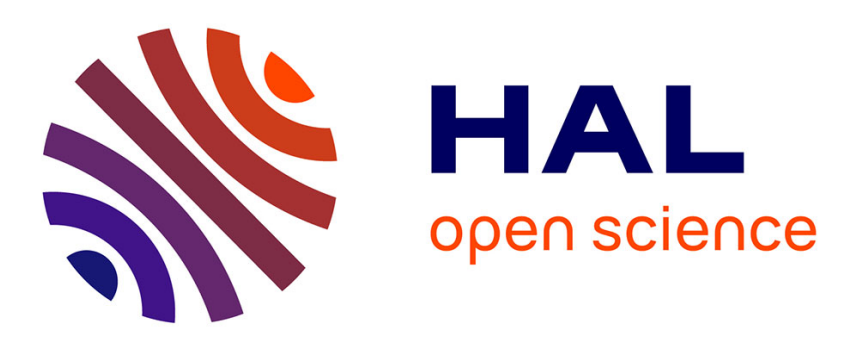

\title{
Terahertz pulse generation by multi-color laser fields with linear versus circular polarization
}

\author{
Alexandre Stathopulos, Stefan Skupin, Luc Bergé
}

\section{To cite this version:}

Alexandre Stathopulos, Stefan Skupin, Luc Bergé. Terahertz pulse generation by multi-color laser fields with linear versus circular polarization. Optics Letters, 2021, 46 (23), pp.5906. 10.1364/OL.442519 . hal-03442239

\section{HAL Id: hal-03442239 \\ https://hal.science/hal-03442239}

Submitted on 23 Nov 2021

HAL is a multi-disciplinary open access archive for the deposit and dissemination of scientific research documents, whether they are published or not. The documents may come from teaching and research institutions in France or abroad, or from public or private research centers.
L'archive ouverte pluridisciplinaire HAL, est destinée au dépôt et à la diffusion de documents scientifiques de niveau recherche, publiés ou non, émanant des établissements d'enseignement et de recherche français ou étrangers, des laboratoires publics ou privés. 


\title{
Terahertz pulse generation by multi-color laser fields with linear versus circular polarization
}

\author{
Alexandre Stathopulos ${ }^{1,2,}$, , Stefan Skupin ${ }^{3}$, and Luc Bergé ${ }^{1,2}$ \\ ${ }^{1}$ CEA, DAM, DIF, F-91297 Arpajon, France \\ 2 Université Paris-Saclay, CEA, LMCE, 91680 Bruyères-le-Châtel, France \\ ${ }^{3}$ Institut Lumière Matière, UMR 5306 Université Lyon 1 - CNRS, Université de Lyon, 69622 Villeurbanne, France \\ *Corresponding author: alexandre.stathopulos@cea.fr
}

We study the influence of the polarization state of multicolor femtosecond laser pulses ionizing air or noble gases on the emitted terahertz radiation. A local-current model and plane-wave evaluations predict a cross-over in the THz energy yields with increasing number of pump harmonics, for which circular laser polarization is more efficient for few harmonics and linear polarization is favourable for more than six pump colors. Comprehensive 3D numerical simulations of gas jet experiments confirm this property for singly- and multiplyionized gases. Rotation of the THz polarization ellipse in the case of circular laser polarization is explained by phase shifts that may alter the phase angle between the harmonics. () 2021 Optical Society of America

http://dx.doi.org/10.1364/ao.XX.XXXXXX

$\mathrm{THz}$ radiation refers to the range of electromagnetic waves associated with $\sim 0.01-10$ ps-scaled oscillation periods, nowadays covered by emitter devices employing ultrafast lasers [1]. Their applications are numerous, spanning from medicine with cancer detection [2], remote identification [3], to greenhouse gas detection and plant life monitoring [4]. Besides overcoming water absorption in atmospheric remote spectroscopy, the need of intense $\mathrm{THz}$ fields is in constant increase, as the most recent applications require high field strengths, e.g., to probe the dynamics of water molecules [5], to drive matter into metastable states [6], or for atom probe tomography [7].

An efficient method to generate intense $\mathrm{THz}$ radiations consists in focusing a two-color ultrashort light pulse composed of the fundamental (FH) and its second harmonic (SH) frequency into a gas. Near focus the pulse creates an electron plasma that produces, through photocurrents, low frequencies belonging to the $\mathrm{THz}$ domain. Such is the case with air or noble gases ionized by two-color laser pulses with $\sim 10^{14} \mathrm{~W} \mathrm{~cm}^{-2}$ intensities, which can radiate broadband, strong $(\geq 0.1 \mathrm{GV} / \mathrm{m}) \mathrm{THz}$ fields with $\sim \mu \mathrm{J}$ energies [8]. This technique has several advantages, as it is free of any material damage and can benefit from the laser filamentation process to produce terahertz pulses remotely [9]. Let us recall that much higher $\mathrm{THz}$ energies $\sim 1-10 \mathrm{~mJ}$ can be obtained by relativistic laser gas-jet $[10,11]$ and laser solid interactions $[12,13]$, but they need TW to PW laser systems delivering
Joule energies and intensities larger than $10^{18} \mathrm{~W} \mathrm{~cm}^{-2}$.

At moderate laser intensities $\sim 10^{14-15} \mathrm{~W} / \mathrm{cm}^{2}$, several techniques to increase the $\mathrm{THz}$ yield by photocurrents have been reported. Using longer FH wavelengths from 0.8 to $2.2 \mu \mathrm{m}$ can already augment the radiated energy by one order of magnitude $[14,15]$. The recent advent of sub-mJ, ultrafast mid-IR $(3.9 \mu \mathrm{m})$ and $\mathrm{CO}_{2}(10.6 \mu \mathrm{m})$ lasers also allowed to reach records of $\sim 2 \%$ in conversion efficiency and $\sim 0.1 \mathrm{~mJ} \mathrm{THz}$ energies for pump energies $<10 \mathrm{~mJ}[16,17]$. Alternative ways may employ gases with higher ionization energies, trigger multiple ionization events [18], or play on the pump duration and plasma dimensions [19].

For a given $\mathrm{FH}$ wavelength, higher $\mathrm{THz}$ performances were attained by increasing the number of colors [20-22] or modifying the polarization state of the $\mathrm{FH}$ and $\mathrm{SH}$ components [23, 24]. On the one hand, Gonzalez de Alaiza Martinez et al. [20] suggested that, with multiple-color pulses arranged in sawtoothlike wave-shape, THz energy could be dramatically increased by a factor $\sim 10$ at constant ionization yield compared with a classical two-color arrangement. On the other hand, Meng et al. [24] experimentally evidenced from helium gas jets that circularly-polarized two-color pulses with same helicity (CP-S) could deliver $\sim 5$ times higher $\mathrm{THz}$ powers than their linearlypolarized, parallel (LP-P) counterparts. Dai et al. [23] earlier reported $\mathrm{THz}$ field polarization remaining linear in CP-S pump configuration, with power invariant to any change in the FH-SH relative phase. Meanwhile, the effects of the pump polarization states were also investigated numerically $[25,26]$. Increase in the $\mathrm{THz}$ power was attributed in [27] to a direct dependency of the drift velocity acquired by photo-ionized electrons on the dominant FH pump amplitude and longer ionization sequences.

Combining the two previous techniques remains to be explored and may considerably increase the energy of the emitted $\mathrm{THz}$ pulses. In the present Letter we investigate $\mathrm{THz}$ performances of pulse profiles containing up to 10 harmonics (or colors) being either linearly or circularly polarized. We report, by means of an analytical approach and local-current (LC) estimates, a major change in the radiated $\mathrm{THz}$ energies when passing from 2 to more than 6 harmonics between LP-P and CP-S sawtoothlike pump pulses at saturated ionization yield. LP-P pulses with large enough number of colors deliver the highest energy yields compared with their CP-S counterpart. This is a generic feature for singly-ionized air molecules or multiply-ionized noble gases 

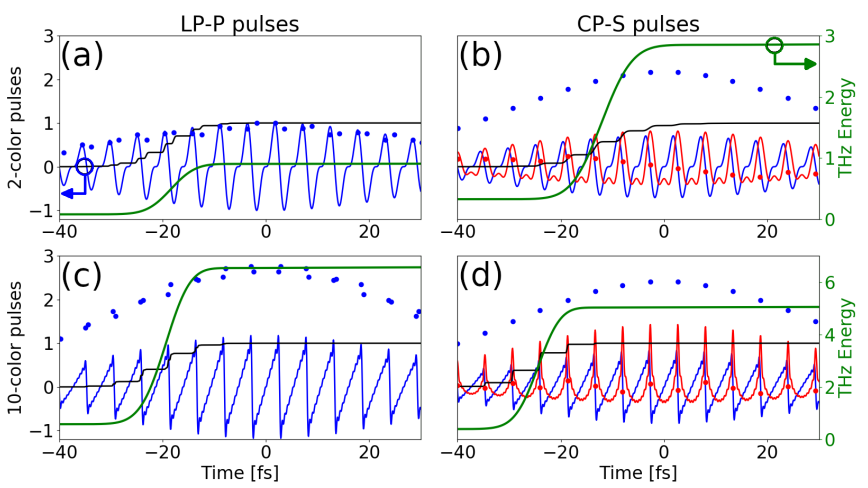

Fig. 1. Left-hand side axis: normalized electron density $N_{e}$ (black curves), laser field components $E_{x, y}$ (blue and red curves), electron drift velocity at ionization times (blue and red dots) as a function of time for 2-color (a) LP-P; (b) CP-S pulses, and 10-color (c) LP-P and (d) CP-S pulses ionizing $\mathrm{O}_{2}$ molecules with $200 \mathrm{TW} / \mathrm{cm}^{2}$ intensity, $1600 \mathrm{~nm}$ FH with $60 \mathrm{fs}$ FWHM [see Eq. (2)]. Right-hand side axis: THz energy (green curves). All quantities are normalized with respect to their maximum value achieved in the 2-color LP-P configuration.

able to produce few tens of $\mu \mathrm{J}$ radiated energies with modest laser pulse parameters. Rotation of the $\mathrm{THz}$ polarization ellipse for CP-S pump configurations is finally addressed and identified as phase shifts induced by nonlinear propagation effects.

According to the LC model [28] and assuming negligible contributions from the Kerr response, the THz emission is proportional to the time derivative of the current density $\vec{J}$ :

$$
\partial_{t} \vec{J}+v_{c} \vec{J}=\frac{e^{2}}{m_{e}} N_{e} \vec{E},
$$

where $e$ and $m_{e}$ are the electron charge and mass, respectively, and $v_{c}=1 / 350 \mathrm{fs}^{-1}$ denotes the electron-neutral collision rate. The growth in the free electron density $N_{e}$ from neutrals' density $N_{a}=2.7 \times 10^{19} \mathrm{~cm}^{-3}$ is, at leading order, modeled by the electron source equation $\partial_{t} N_{e}=W(E)\left(N_{a}-N_{e}\right)$, where $W(E)$ is the ionization rate only depending on $E(t) \equiv\left|\vec{E}_{L}(t)\right|$. This rate is given, in a first approximation, by the quasi-static tunneling (QST) rate, $W[E(t)]=(\alpha / E(t)) \mathrm{e}^{-\beta / E(t)}$, where the constants $(\alpha, \beta)$ can be found in [28]. It is applied to the $\mathrm{O}_{2}$ molecules of air $\left(20 \%\right.$ of $\left.N_{a}\right)$ with ionization potential $U_{i}=12.1 \mathrm{eV}$ irradiated by the transverse vectorial Gaussian multi-color field:

$$
\vec{E}_{L}(t)=E_{0} \sum_{j=1}^{N} \frac{\mathcal{E}_{j}}{2^{\rho / 2}} \mathrm{e}^{-2 \ln 2 \frac{t^{2}}{\tau_{j}^{2}}}\left(\begin{array}{c}
\sin \left[j \omega_{0} t+\varphi_{j}\right] \\
\rho \cos \left[j \omega_{0} t+\varphi_{j}\right]
\end{array}\right),
$$

where $\mathcal{E}_{j}=(-1)^{j+1} /\left(j \sqrt{\sum_{k=1}^{N} k^{-2}}\right)$ for sawtooth-shaped input fields composed of $N$ colors and $\mathcal{E}_{1}=\sqrt{1-r}, \mathcal{E}_{2}=\sqrt{r}$ for two-color setups in LP-P configuration for which $\rho=0$. CP-S pulses are similarly described with $\rho=1$. The parameter $r$, here always fixed to 0.2 , is the two-color $\mathrm{SH}$ intensity fraction. The phase $\varphi_{j}$ is a constant phase shift. We shall assume same FHWM duration $\tau_{j}=60 \mathrm{fs}$ for all colors if not stated otherwise.

In the plane wave approximation, quasi-DC components of the photocurrents produced by two-color fields $(N=2)$ lead to $\mathrm{THz}$ fields behaving as $\vec{E}_{\mathrm{THz}} \propto(\cos \phi, 0)^{T}$ in LP-P and $\vec{E}_{\mathrm{THz}} \propto(\cos \phi, \sin \phi)^{T}$ in CP-S [27], where $\phi=\varphi_{2}-2 \varphi_{1}$ is the effective phase angle between $\mathrm{FH}$ and $\mathrm{SH}$. This simple expression justifies the expected linear polarization of $\mathrm{THz}$ radiation and invariance of the $\mathrm{THz}$ energy with respect to $\phi$ when using CP-S pump pulses. Following [28], the local THz electric field can be evaluated by the low-frequency filtering of $\partial_{t} \vec{J}$ [Eq. (1)] in which $N_{e}(t)$ increases stepwise through elementary steps $\delta N_{n}$ at the ionization times $t=t_{n}$ defined by the extrema of the laser electric field. By using $N_{e}(t)=\sum_{n} \delta N_{n} H_{n}\left(t-t_{n}\right)$ where $H_{n}(t)$ is close to the Heaviside function, the current density expresses as $\vec{J}(t)=-e \sum_{n} \delta N_{n}\left[\vec{v}_{f}(t)-\vec{v}_{f}\left(t_{n}\right) \mathrm{e}^{-v_{c}\left(t-t_{n}\right)}\right] H_{n}\left(t-t_{n}\right)$ where $\vec{v}_{f}(t)=-\left(e / m_{e}\right) \int_{-\infty}^{t} \vec{E}_{L}\left(t^{\prime}\right) \mathrm{e}^{-v_{c}\left(t-t^{\prime}\right)} d t^{\prime}$ describes the net drift velocity which free electrons born at $t=-\infty$ would acquire from $E_{L}$. The low-frequency (second) term of this expression highlights that electron acceleration at $t=t_{n}$ is proportional to $v_{f}\left(t_{n}\right)$. Approximated in the limits $\omega / \omega_{0}, v_{c} / \omega_{0} \rightarrow 0$ [20], this term provides an estimate of the THz energy as $\eta_{\mathrm{THz}} \propto\left[\sum_{n} \delta N_{n} v_{f}\left(t_{n}\right)\right]^{2}$. This approach is expected to supply qualitative features of direct numerical integrations of Eq. (1), which in turn supplies approximate results to $3 \mathrm{D}$ comprehensive propagation models.

Figure 1 displays examples of field patterns and their $\mathrm{THz}$ yields for two- and 10-color, 60-fs Gaussian pulses with 200 $\mathrm{TW} / \mathrm{cm}^{2}$ intensity ionizing $\mathrm{O}_{2}$ with $\mathrm{FH}$ wavelength $\lambda_{0} \equiv$ $2 \pi c / \omega_{0}=1600 \mathrm{~nm}$. The blue (red) curve refers to the $x$ (resp. $y)$ component of the laser electric field. With $\varphi_{j}=0$ the relative phase between harmonics in Eq. (2) promotes an optimallyemitting plasma in the LP-P case [20]. THz yields are computed for a $90-\mathrm{THz}$ large frequency window. Comparing the 2-color configurations [Figs. 1(a,b)], the THz energy curve numerically computed from Eq. (1) appears larger in the CP-S case compared with the LP-P one. Both pulse configurations fully ionize $\mathrm{O}_{2}$ to $\mathrm{O}_{2}^{+}$. However, even though the CP-S pulse reaches saturation later in time, the electron drift velocity $v_{f}\left(t_{n}\right)$ acquired at each ionization instant $t_{n}$ (see blue dots) is much higher for CP-S than for LP-P pulses, explaining the superiority of the CP-S pulses. Surprisingly, an opposite conclusion follows for a 10-color sawtooth pulse $(N=10)$ as the LP-P configuration yields more $\mathrm{THz}$ energy than the CP-S one. Here, the contribution from $v_{f}\left(t_{n}\right)$ is more important in the LP-P case compared with the CP-S one.

To clear up this inversion of performances, Fig. 2(a) details the THz yield numerically evaluated from the LC model by increasing the number of harmonics in Eq. (2). Trends in the radiated energy indeed become reverted between LP-P and CP-S pulses for a number of colors close to 6 . This property can directly be found from the analytical expressions $\eta_{\mathrm{THz}}^{\mathrm{LP}-\mathrm{P}} \sim$ $\left(\sum_{j=1}^{N} \cos (j \pi /(N+1)) / j^{2}\right)^{2}$ and $\eta_{\mathrm{THz}}^{\mathrm{CP}-\mathrm{S}} \sim \frac{1}{2}\left(\sum_{j=1}^{N} 1 / j^{2}\right)^{2}$, assuming constant ionization steps $\delta N_{n}$ and same maximum electron density. The former constraint overestimates the photocurrent efficiency over each electron density step for $N>2$ and CP-S configurations. The latter one is easily satisfied for 200 $\mathrm{TW} / \mathrm{cm}^{2}$ intensity using the QST tunnel rate because ionization saturates. It is also fulfilled for other ionization rates, e.g., the Perelomov-Popov-Terent'ev (PPT) rate [29] with effective charge numbers $Z_{\mathrm{O}_{2}}^{*} \sim 1$ [see Fig 2(b)]. In contrast, for $Z_{\mathrm{O}_{2}}^{*}=0.53$ [30], the ionization yield strongly varies below saturation between the two pump polarizations and our analytical estimates no longer hold.

The previous properties are now checked by direct 3D numerical computations based on a vectorial version of the unidirectional pulse propagation equation (UPPE) [31]. This numerical model governs the forward-propagating transverse electric field components $E_{x}, E_{y}$ of elliptically-polarized pulses subject to linear dispersion and diffraction, together with third-order 

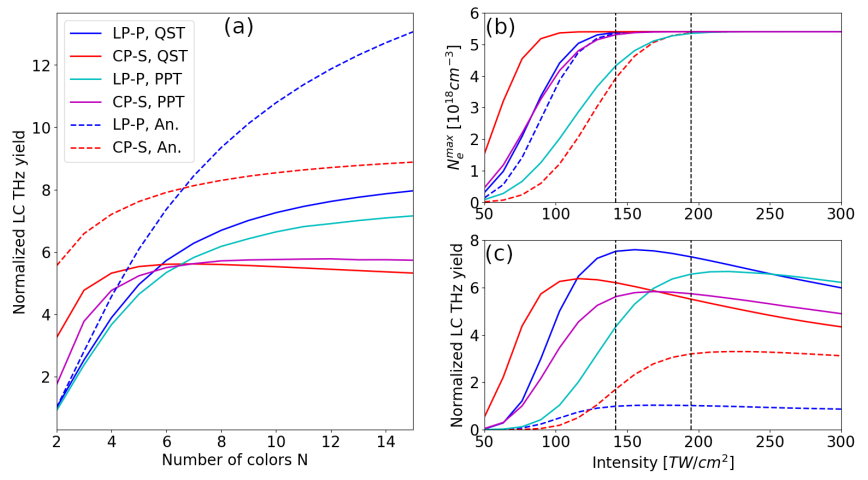

Fig. 2. (a) THz energy yields normalized to their LP-P twocolor counterpart and computed from the LC model using Eq. (2) for unchirped 2- and 10-color 60-fs pulses with QST ionization rate (blue/red) and 10-color pulses undergoing the PPT rate with $Z_{\mathrm{O}_{2}}^{*}=1$ (purple/cyan curves). Dashed curves refer to analytical expressions $\left(\eta_{\mathrm{THz}}\right)$ also normalized to the LP-P 2-color case (see text). (b,c) Peak electron density and conversion efficiency as functions of the input intensity. Vertical dotted curves indicate the minimum intensity value from which singly-ionized charge states are completed.

nonlinear polarization, photo-ionization and related losses [32]. More details about this vectorial model can be found in [27] while the physical parameters for air are taken from [33]. We first validate our theoretical expectations using the simple QST model for singly-ionizable oxygen $\left(20 \%\right.$ of $\left.N_{a}\right)$ at input intensity $\sim 200 \mathrm{TW} / \mathrm{cm}^{2}$. Figures 3(a,b) display for this configuration an ionization yield clamped to saturation during early propagation with two and ten colors. Results from Fig. 2 should thus apply and, indeed, we observe that, unlike the basic 2-color configuration, $\mathrm{THz}$ energy increases more in LP-P polarization with 10 colors [Fig. 3(b)]. In Fig. 3(a) fluence patterns reveal a net conical emission for two colors at $z=500 \mu \mathrm{m}$ forming an angle close to $\sim 4-5^{\circ}$ in agreement with Yu et al. [34]. Conical emission here occurs earlier for LP-P pulses $\left(\sim 5^{\circ}\right)$ compared to CP-S ones $\left(\sim 4^{\circ}\right)$, from the middle of the optical path. Similar features follow from a more realistic ionization model for air [30], encompassing ionization of both $\mathrm{O}_{2}\left(20 \%, Z_{\mathrm{O}_{2}}^{*}=0.53\right)$ and $\mathrm{N}_{2}(80 \%$, $\left.Z_{N_{2}}^{*}=0.9\right)$ molecules with a PPT rate by 10-color pulses [Figs. $3(\mathrm{c}, \mathrm{d})]$. Note the sudden increase in the electron density driven by the LP-P pulse, which follows from local phase shifts between the harmonics strongly affecting the peak electric field value (see inset). This enhances the ionization response at $z \approx 20 \mu \mathrm{m}$ and the electron density accordingly. Consequently, this pulse configuration takes over its CP-S counterpart along the remaining propagation range. Interestingly, conical emission patterns with 10 colors remain comparable with those produced by two colors [compare Figs. 3(a) and 3(d)]. Finally, we also tested in Figs. $3(\mathrm{e}, \mathrm{f})$ a multi-ionization (ADK-driven) module detailed in [35] for gas jets of argon (blue/red curves) and of helium (purple curve) at $0.2 \mathrm{~atm}$ pressure $\left(N_{a}=5.4 \times 10^{18} \mathrm{~cm}^{-3}\right)$ and for different pump energies. It is clear that, even with higher charge states $N_{e} / N_{a}$ close to 2 , the major tendencies remain, i.e., LP-P pulses with $400 \mathrm{TW} / \mathrm{cm}^{2}$ intensity in argon deliver $\mathrm{THz}$ radiation with highest energy, close to $\sim 10 \mu \mathrm{J}$. Helium with larger ionization potentials promote the highest yields, above $40 \mu \mathrm{J}$, reached with $5 \mathrm{PW} / \mathrm{cm}^{2}$ intensity. We checked that accounting for avalanche ionization does not change these qualitative behaviors.

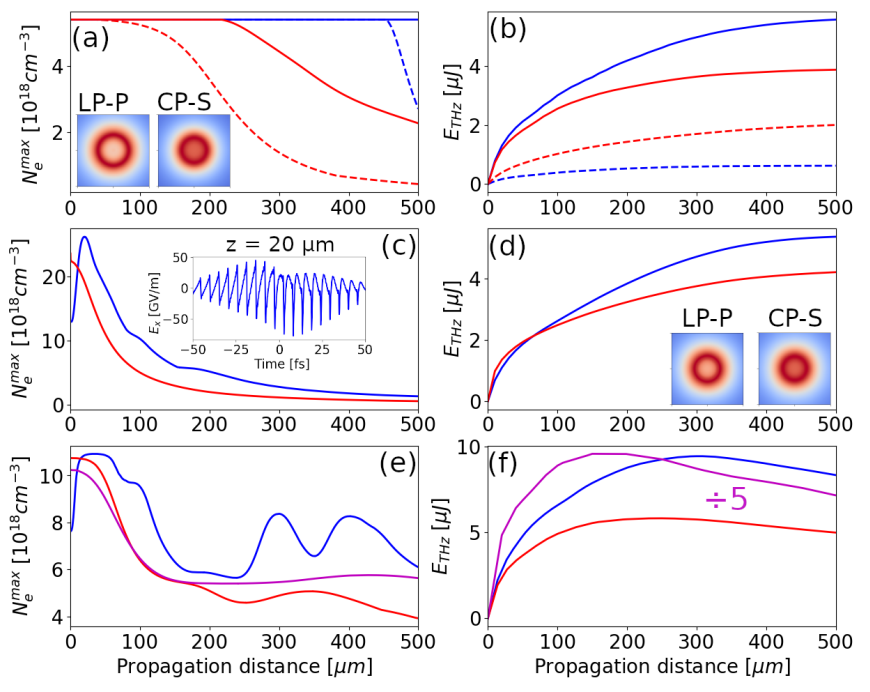

Fig. 3. $(\mathrm{a}, \mathrm{c}, \mathrm{e})$ Peak electron densities and $(\mathrm{b}, \mathrm{d}, \mathrm{f}) \mathrm{THz}$ energies computed with a 3D UPPE code for 2-color (dashed curves) and 10-color (solid curves) pulses. Blue/red curves stand for LP-P/CP-S configurations. $(\mathrm{a}, \mathrm{b}) \mathrm{O}_{2}$ ionized with a QST rate. (c,d) Realistic air with $20 \% \mathrm{O}_{2}$ and $80 \% \mathrm{~N}_{2}\left(U_{i}=15.6 \mathrm{eV}\right)$ ionized with PPT rate using Talebpour et al.'s charge numbers $\mathrm{Z}_{\mathrm{O}_{2}}^{*}=0.53, \mathrm{Z}_{\mathrm{N}_{2}}^{*}=0.9$ [30]. (e,f) 10-color-driven multiplyionized argon (blue/red curves) and helium in CP-S configuration (purple curve) at $0.2 \mathrm{~atm}$ pressure employing the multiionization scheme of [18]. The LP-P pulse triggers density ratio $N_{e} / N_{a}$ varying from 1.41 to 2.02 ; CP-S pulses achieve density ratios of 1.99 and 1.89 in $\mathrm{Ar}$ and $\mathrm{He}$, respectively. In (a) and (d) fluence patterns illustrate conical emissions at $z=500 \mu \mathrm{m}$ in the transverse plane $-100 \leq x, y \leq 100 \mu \mathrm{m}$.

Finally, visual inspection of the UPPE3D results revealed a generic rotation of the $\mathrm{THz}$ polarization angle along $0.5 \mathrm{~mm}$ propagation ranges for the CP-S pump configuration. As recalled above, in a 2-color CP-S setup the $\mathrm{THz}$ polarization angle is determined by the effective phase angle between the two harmonics. Figure 4(a) reports in this respect an anticlockwise rotation that continuously follows the growth of $\mathrm{THz}$ emission [see Fig. 3(b)] for singly-ionizable oxygen (20\% of $N_{a}$, QST rate). As observed from Fig. 4(b) the same property occurs with 10 colors, i.e., the $\mathrm{THz}$ radiation globally remains almost linearly polarized, but with slightly larger rotation angle. After testing several potential processes causing this rotation, we inferred that nonlinear propagation effects (e.g., plasma dispersion, distortions induced by local plasma defocusing etc.) introduce phase shifts into the harmonics that cannot preserve a constant relative phase along the pulse oscillations [see Fig. 4(c) detailing FH and $\mathrm{SH}$ components of a 2-color pulse at $z=500 \mu \mathrm{m}]$. This can be approached by considering a time dependent phase function $\varphi_{j}(t)$ including now linear or quadratic chirp contributions. Figure 4(d) tends to confirm this property when one evaluates from the LC model the $\mathrm{THz}$ field produced by a Gaussian pulse acquiring different phase shifts, e.g., from a constant phase angle to linear and quadratic chirps that break the constancy of the 2 -color relative phase for equal pulse durations $\tau_{2}=\tau_{1}$.

In conclusion we numerically explored the possibility to enhance the THz energy yield by multi-colored sawtooth pulses arranged in optimum polarization states. Surprisingly, circularlypolarized pulses with 6 or more harmonics do not maximize 

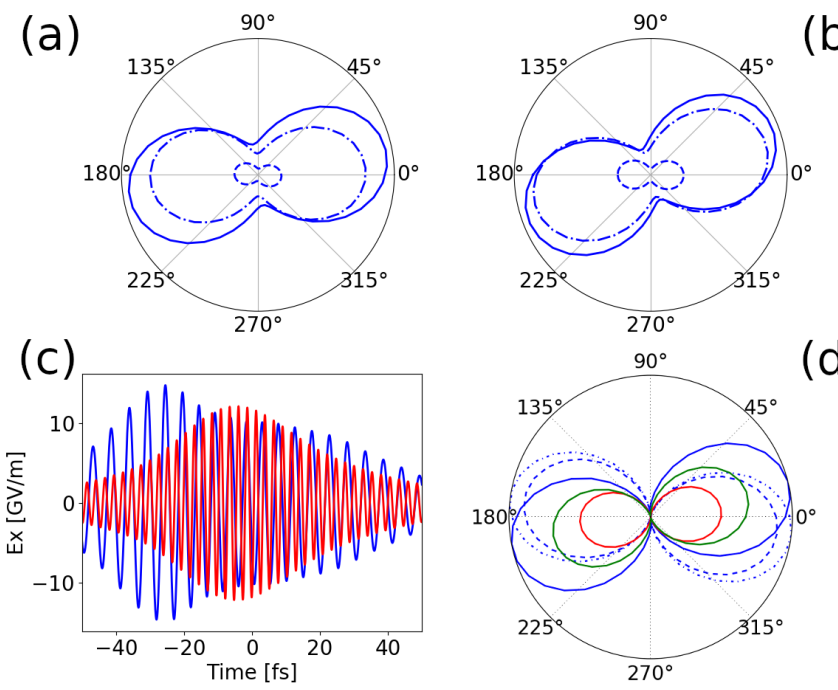

Fig. 4. (a) Angular polarization diagrams (energy flux transmitted by a polarizer) extracted from UPPE3D simulations for 2-color Gaussian pulses at the distances: $z=10 \mu \mathrm{m}$ (dashed), $z=250 \mu \mathrm{m}$ (dash-dotted) and $z=500 \mu \mathrm{m}$ (solid curve); (b) same information for a 10-color sawtooth pulse; (c) FH and SH components corresponding to (a) at $z=500 \mu \mathrm{m}$ and displaying a chirped phase. (d) Polarization rotation induced by a constant phase angle $\phi=0.19$ rad (red curve) reproducing (a), by a linear chirp with $\varphi_{j}(t)=-1.38 t / \tau_{j}$ (green curve) and by a quadratic chirp with $\varphi_{j}(t)=5.54 t^{2} / \tau_{j}^{2}$ in Eq. (2) for two colors with various pulse durations: no chirp (dashed), $\tau_{2}=\tau_{1} / \sqrt{2}$ (dash-dotted) and $\tau_{2}=\tau_{1}$ (solid blue curve).

the $\mathrm{THz}$ energy performances, as linearly-polarized pulses still supply the highest electron density, from singly- or multiplyionized species. LP-P pulses appear more sensitive to local phase shifts that increase the ionization yield. Our analysis provided evidence that multiple color arrangements deliver the highest energy yields, up to $50 \mu \mathrm{J}$ in He at moderate intensities, in a more efficient way than changing the pump polarization state. However, for few-color sources, passing to circularly polarized pumps (CP-S) remains beneficial for the THz yield. We finally attributed the rotation of the $\mathrm{THz}$ field for CP-S pump polarization to the introduction of phase shifts between the harmonics along propagation. With the increasing progress in combining various laser frequencies, we believe that our results will be useful in the all-optical generation of strong $\mathrm{THz}$ fields and their applications.

\section{FUNDING}

Agence Nationale de la Recherche (ANR) (ALTESSE: ANR-19ASMA-0007); Grand Équipement National De Calcul Intensif (GENCI) (A0060507594); Qatar National Research Fund (NPRP 12S-0205-190047).

\section{DISCLOSURES}

The authors declare no conflicts of interest.

\section{REFERENCES}

1. M. Tonouchi, Nat. Photon. 1, 97 (2007).

2. C. Yu, S. Fan, Y. Sun, and E. Pickwell-Macpherson, Quant. Imaging, Med. Surg. 2, 33 (2012).
3. L. Bergé, K. Kaltenecker, S. Engelbrecht, A. Nguyen, S. Skupin, L. Merlat, B. Fischer, B. Zhou, I. Thiele, and P. U. Jepsen, Eur. Phys. Lett. 126, 24001 (2019).

4. R. Gente and M. Koch, Plant Methods 11, 15 (2015).

5. F. Novelli, B. Guchhait, and M. Havenith, Materials 13, 1311 (2021).

6. X. Li, T. Qiu, J. Zhang, E. Baldini, J. Lu, A. M. Rappe, and K. A. Nelson, Science 364, 1079 (2019).

7. A. Vella, J. Houard, L. Arnoldi, M. Tang, M. Boudant, A. Ayoub, A. Normand, G. D. Costa, and A. Hideur, Sci. Adv. 7, eabd7259 (2021).

8. K. Y. Kim, A. J. Taylor, J. H. Glownia, and G. Rodriguez, Nat. Photon. 2, 605 (2008).

9. L. Bergé, S. Skupin, C. Köhler, I. Babushkin, and J. Herrmann, Phys. Rev. Lett. 110, 073901 (2013).

10. W. P. Leemans, C. Geddes, J. Faure, C. Tóth, J. van Tilborg, C. B. Schroeder, E. Esarey, G. Fubiani, D. Auerbach, B. Marcelis, M. A. Carnahan, R. A. Kaindl, J. Byrd, and M. C. Martin, Phys. Rev. Lett. 91, 074802 (2003).

11. J. Déchard, A. Debayle, X. Davoine, L. Gremillet, and L. Bergé, Phys. Rev. Lett. 120, 144801 (2018).

12. G. Liao, Y. Li, and L. et al., Proc. Nat. Ac. Sci. 116, 3994 (2019).

13. J. Déchard, X. Davoine, L. Gremillet, and L. Bergé, Phys. Plasmas 27, 093105 (2020).

14. M. Clerici, M. Peccianti, B. E. Schmidt, L. Caspani, M. Shalaby, M. Giguère, A. Lotti, A. Couairon, F. Légaré, T. Ozaki, D. Faccio, and R. Morandotti, Phys. Rev. Lett. 110, 253901 (2013).

15. A. Nguyen, K. J. Kaltenecker, J.-C. Delagnes, B. Zhou, E. Cormier, N. Fedorov, R. Bouillaud, D. Descamps, I. Thiele, S. Skupin, P. U. Jepsen, and L. Bergé, Opt. Lett. 44, 1844 (2019).

16. A. Nguyen, P. G. de Alaiza Martínez, I. Thiele, S. Skupin, and L. Bergé, Phys. Rev. A 97, 063839 (2018).

17. A. D. Koulouklidis and C. Gollner and V. Shumakova and V. Yu. Fedorov and A. Pugžlys and A. Baltuška and S. Tzortzakis, Nat. Commun. 11, 292 (2020).

18. A. Debayle, P. González de Alaiza Martínez, L. Gremillet, and L. Bergé, Phys. Rev. A 91, 041801 (2015).

19. F. Buccheri and X.-C. Zhang, Optica 2, 366 (2015).

20. P. González de Alaiza Martínez, I. Babushkin, L. Bergé, S. Skupin, E. Cabrera-Granado, C. Köhler, U. Morgner, A. Husakou, and J. Herrmann, Phys. Rev. Lett. 114, 183901 (2015).

21. B. Zhou, Y. Wang, L. Hong, D. Mahdi, and P. U. Jepsen, 2018 43rd Int. Conf. Infrared, Millim. Terahertz Waves (IRMMW-THz, IEEE 2018) pp. 1-1 (2018).

22. V. Vaičaitis, O. Balachninaite, U. Morgner, and I. Babushkin, J. Appl. Phys. 125, 173103 (2019).

23. J. Dai, N. Karpowicz, and X.-C. Zhang, Phys. Rev. Lett. 103, 023001 (2009).

24. C. Meng, W. Chen, X. Wang, Z. Lü, Y. Huang, J. Liu, D. Zhang, Z. Zhao, and J. Yuan, Appl. Phys. Lett. 109, 131105 (2016).

25. V. Y. Fedorov, A. D. Koulouklidis, and S. Tzortzakis, Plasmas Phys. Cont. Fusion 59, 014025 (2017).

26. O. Kosareva, M. Esaulkov, N. Panov, V. Andreeva, D. Shipilo, P. Solyankin, A. Demircan, I. Babushkin, V. Makarov, U. Morgner, A. Shkurinov, and A. Savelev, Opt. Lett. 43, 90 (2018).

27. C. Tailliez, A. Stathopulos, S. Skupin, D. Buožius, I. Babushkin, V. Vaičaitis, and L. Bergé, New J. Phys. 22, 103038 (2020).

28. I. Babushkin, S. Skupin, A. Husakou, C. Köhler, E. Cabrera-Granado, L. Bergé, and J. Herrmann, New J. Phys. 13, 123029 (2011).

29. A. M. Perelomov, V. S. Popov, and M. V. Terent'ev, Sov. Phys. JETP 23, 924 (1966).

30. A. Talebpour, J. Yang, and S. L. Chin, Opt. Commun. 163, 29 (1999).

31. M. Kolesik and J. V. Moloney, Phys. Rev. E 70, 036604 (2004).

32. L. Bergé, S. Skupin, R. Nuter, J. Kasparian, and J. P. Wolf, Rep. Prog. Phys. 70, 1633 (2007).

33. A. Nguyen, P. G. de Alaiza Martínez, J. Déchard, I. Thiele, I. Babushkin, S. Skupin, and L. Bergé, Opt. Express 25, 4720 (2017).

34. Y. S. You, T. I. Oh, and K. Y. Kim, Phys. Rev. Lett. 109, 183902 (2012).

35. A. Debayle, P. González de Alaiza Martínez, L. Gremillet, and L. Bergé, Phys. Rev. A 91, 041801 (2015). 


\section{FULL REFERENCES}

1. M. Tonouchi, "Cutting-edge terahertz technology," Nat. Photon. 1, 97 (2007).

2. C. Yu, S. Fan, Y. Sun, and E. Pickwell-Macpherson, "The potential of terahertz imaging for cancer diagnosis: A review of investigations to date," Quant. Imaging, Med. Surg. 2, 33-45 (2012).

3. L. Bergé, K. Kaltenecker, S. Engelbrecht, A. Nguyen, S. Skupin, L. Merlat, B. Fischer, B. Zhou, I. Thiele, and P. U. Jepsen, "Terahertz spectroscopy from air plasmas created by two-color femtosecond laser pulses: The ALTESSE project," Eur. Phys. Lett. 126, 24001 (2019).

4. R. Gente and M. Koch, "Monitoring leaf water content with $\mathrm{THz}$ and sub-THz waves," Plant Methods 11, 15 (2015).

5. F. Novelli, B. Guchhait, and M. Havenith, "Towards intense thz spectroscopy on water: Characatrization of optical rectification by gap, oh1, and dstms at opa wavelengths," Materials 13, 1311 (2021).

6. X. Li, T. Qiu, J. Zhang, E. Baldini, J. Lu, A. M. Rappe, and K. A. Nelson, "Terahertz field-induced ferroelectricity in quantum paraelectric srtio 3 ," Science 364, 1079 (2019).

7. A. Vella, J. Houard, L. Arnoldi, M. Tang, M. Boudant, A. Ayoub, A. Normand, G. D. Costa, and A. Hideur, "High-resolution terahertz-driven atom probe tomography," Sci. Adv. 7, eabd7259 (2021).

8. K. Y. Kim, A. J. Taylor, J. H. Glownia, and G. Rodriguez, "Coherent control of terahertz supercontinuum generation in ultrafast laser-gas interactions," Nat. Photon. 2, 605 (2008).

9. L. Bergé, S. Skupin, C. Köhler, I. Babushkin, and J. Herrmann, "3D numerical simulations of $\mathrm{THz}$ generation by two-color laser filaments," Phys. Rev. Lett. 110, 073901 (2013).

10. W. P. Leemans, C. Geddes, J. Faure, C. Tóth, J. van Tilborg, C. B. Schroeder, E. Esarey, G. Fubiani, D. Auerbach, B. Marcelis, M. A. Carnahan, R. A. Kaindl, J. Byrd, and M. C. Martin, "Observation of terahertz emission from a laser-plasma accelerated electron bunch crossing a plasma-vacuum boundary," Phys. Rev. Lett. 91, 074802 (2003).

11. J. Déchard, A. Debayle, X. Davoine, L. Gremillet, and L. Bergé, "Terahertz Pulse Generation in Underdense Relativistic Plasmas: From Photoionization-Induced Radiation to Coherent Transition Radiation," Phys. Rev. Lett. 120, 144801 (2018).

12. G. Liao, Y. Li, and L. et al., "Multimillijoule coherent terahertz bursts from picosecond laser-irradiated metal foils," Proc. Nat. Ac. Sci. 116, 3994 (2019)

13. J. Déchard, X. Davoine, L. Gremillet, and L. Bergé, "Terahertz emission from submicron solid targets irradiated by ultraintense femtosecond laser pulses," Phys. Plasmas 27, 093105 (2020).

14. M. Clerici, M. Peccianti, B. E. Schmidt, L. Caspani, M. Shalaby, M. Giguère, A. Lotti, A. Couairon, F. Légaré, T. Ozaki, D. Faccio, and R. Morandotti, "Wavelength scaling of terahertz generation by gas ionization," Phys. Rev. Lett. 110, 253901 (2013).

15. A. Nguyen, K. J. Kaltenecker, J.-C. Delagnes, B. Zhou, E. Cormier, N. Fedorov, R. Bouillaud, D. Descamps, I. Thiele, S. Skupin, P. U. Jepsen, and L. Bergé, "Wavelength scaling of terahertz pulse energies delivered by two-color air plasmas," Opt. Lett. 44, 1844 (2019).

16. A. Nguyen, P. G. de Alaiza Martínez, I. Thiele, S. Skupin, and L. Bergé, "Broadband terahertz radiation from two-color mid- and far-infrared laser filaments in air," Phys. Rev. A 97, 063839 (2018).

17. A. D. Koulouklidis and C. Gollner and V. Shumakova and V. Yu. Fedorov and A. Pugžlys and A. Baltuška and S. Tzortzakis, "Observation of extremely efficient terahertz generation from mid-infrared two-color laser filaments," Nat. Commun. 11, 292 (2020).

18. A. Debayle, P. González de Alaiza Martínez, L. Gremillet, and L. Bergé, "Non-monotonic increase in laser-driven $\mathrm{THz}$ emissions through multiple ionization events," Phys. Rev. A 91, 041801 (2015).

19. F. Buccheri and X.-C. Zhang, "Terahertz emission from laser-induced micro plasma in ambient air," Optica 2, 366 (2015).

20. P. González de Alaiza Martínez, I. Babushkin, L. Bergé, S. Skupin, E. Cabrera-Granado, C. Köhler, U. Morgner, A. Husakou, and J. Herrmann, "Boosting terahertz generation in laser-field ionized gases using a sawtooth wave shape," Phys. Rev. Lett. 114, 183901 (2015).

21. B. Zhou, Y. Wang, L. Hong, D. Mahdi, and P. U. Jepsen, "High-efficiency sub-single-cycle THz wave generation by three color air plasma," 2018 43rd Int. Conf. Infrared, Millim. Terahertz Waves (IRMMW-THz, IEEE 2018) pp. 1-1 (2018).

22. V. Vaičaitis, O. Balachninaitè, U. Morgner, and I. Babushkin, "Terahertz radiation generation by three-color laser pulses in air filament," J. Appl. Phys. 125, 173103 (2019).

23. J. Dai, N. Karpowicz, and X.-C. Zhang, "Coherent polarization control of terahertz waves generated from two-color laser-induced gas plasma," Phys. Rev. Lett. 103, 023001 (2009).

24. C. Meng, W. Chen, X. Wang, Z. Lü, Y. Huang, J. Liu, D. Zhang, Z. Zhao, and J. Yuan, "Enhancement of terahertz radiation by using circularly polarized two-color laser fields," Appl. Phys. Lett. 109, 131105 (2016).

25. V. Y. Fedorov, A. D. Koulouklidis, and S. Tzortzakis, "THz generation by two-color femtosecond filaments with complex polarization states: four-wave mixing versus photocurrent contributions," Plasmas Phys. Cont. Fusion 59, 014025 (2017).

26. O. Kosareva, M. Esaulkov, N. Panov, V. Andreeva, D. Shipilo, P. Solyankin, A. Demircan, I. Babushkin, V. Makarov, U. Morgner, A. Shkurinov, and A. Savelev, "Polarization control of terahertz radiation from two-color femtosecond gas breakdown plasma," Opt. Lett. 43, 90 (2018).

27. C. Tailliez, A. Stathopulos, S. Skupin, D. Buožius, I. Babushkin, V. Vaičaitis, and L. Bergé, "Terahertz pulse generation by two-color laser fields with circular polarization," New J. Phys. 22, 103038 (2020).

28. I. Babushkin, S. Skupin, A. Husakou, C. Köhler, E. Cabrera-Granado, L. Bergé, and J. Herrmann, "Tailoring terahertz radiation by controling tunnel photoionization events in gases," New J. Phys. 13, 123029 (2011)

29. A. M. Perelomov, V. S. Popov, and M. V. Terent'ev, "Ionization of atoms in an alternating electric field: I," Sov. Phys. JETP 23, 924 (1966).

30. A. Talebpour, J. Yang, and S. L. Chin, "Semi-empirical model for the rate of tunnel ionization of $\mathrm{N}_{2}$ and $\mathrm{O}_{2}$ molecule in an intense Ti:sapphire laser pulse," Opt. Commun. 163, 29 (1999).

31. M. Kolesik and J. V. Moloney, "Nonlinear optical pulse propagation simulation: From Maxwell's to unidirectional equations," Phys. Rev. E 70, 036604 (2004)

32. L. Bergé, S. Skupin, R. Nuter, J. Kasparian, and J. P. Wolf, "Optical ultrashort filaments in weakly-ionized, optically-transparent media," Rep. Prog. Phys. 70, 1633 (2007).

33. A. Nguyen, P. G. de Alaiza Martínez, J. Déchard, I. Thiele, I. Babushkin, S. Skupin, and L. Bergé, "Spectral dynamics of thz pulses generated by two-color laser filaments in air: the role of kerr nonlinearities and pump wavelength," Opt. Express 25, 4720 (2017).

34. Y. S. You, T. I. Oh, and K. Y. Kim, "Off-axis phase-matched terahertz emission from two-color laser-induced plasma filaments," Phys. Rev. Lett. 109, 183902 (2012).

35. A. Debayle, P. González de Alaiza Martínez, L. Gremillet, and L. Bergé, "Non-monotonic increase in laser-driven thz emissions through multiple ionization events," Phys. Rev. A 91, 041801 (2015). 